\title{
Role of mannose-binding lectin in intestinal homeostasis and fungal elimination
}

\author{
L Choteau $^{1,2,3,4}$, M Parny ${ }^{1,2,3}$, N François ${ }^{5}$, B Bertin ${ }^{1,2,3}$, M Fumery ${ }^{1,2,3}$, L Dubuquoy ${ }^{1,2,3}$,

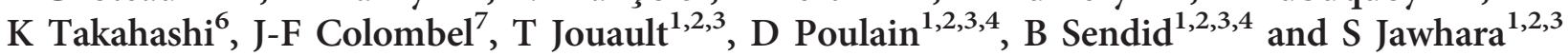

Mannose-binding lectin (MBL) is a soluble lectin of the innate immune system that is produced by the liver and secreted into the circulation where it activates the lectin complement pathway, enhances phagocytosis of microorganisms by leukocytes, and modulates inflammation. MBL can recognize patterns on the surface of different pathogens, including Candida albicans. Our aims were to investigate whether MBL is expressed in the gut epithelium and to examine its effect on the modulation of intestinal inflammation and C. albicans elimination. Using reverse transcriptase-PCR, MBL transcripts were highly expressed in different parts of the mouse gut. MBL expression was also detected by immunoblotting and immunolocalization in response to $C$. albicans colonization of the gut; the highest expression of MBL was detected in the stomach. Blocking MBL by administering mannans to mice increased C. albicans colonization. MBL-deficient mice had a higher level of colonization than wild-type mice. Dextran sodium sulfate-induced colitis promoted C. albicans dissemination to the kidneys and lungs of MBL-deficient mice. MBL-deficient mice exhibited elevated expression of interleukin (IL)-17, IL-23, dectin-1, and Toll-like receptor-4. This study shows that MBL expression is induced in the gut in response to $C$. albicans sensing and is required for intestinal homeostasis and host defense against $C$. albicans.

\section{INTRODUCTION}

Mannose-binding lectin (MBL) is a collectin, produced mainly by hepatocytes, that circulates in multimeric form with a predominance of the quaternary structure. ${ }^{1} \mathrm{MBL}$ forms a complex with three MBL-associated serine proteases (MASP). ${ }^{2}$ MASP1 and MASP2 can be associated on the same complex with MBL. ${ }^{3}$ Human MBL is encoded by a single gene, MBL2, whereas in rodents there are two forms of MBL, MBL-A and MBL-C. ${ }^{4}$ MBL activates the lectin complement pathway after recognition of microorganisms through the carbohydraterecognition domain. The carbohydrate-recognition domain of MBL senses polysaccharide patterns such as D-mannose, L-fucose, and $\mathrm{N}$-acetylglucosamine on several clinically relevant pathogens, including Candida albicans. This results in the activation of MASP1 and MASP2, leading to the complement cascade. $^{5,6}$

C. albicans is an opportunistic fungal pathogen commonly found in the human gastrointestinal (GI) tract, causing the life-threatening disease invasive candidiasis (IC). ${ }^{5,6}$ GI colonization is the predominant source of $C$. albicans infections, which are a serious clinical problem with high morbidity and mortality. ${ }^{7,8}$ MBL is involved in the first line of host defense against IC. Changes in circulating MBL levels are observed during the course of IC, with a dramatic decrease during the 2 days before positive blood cultures. ${ }^{9}$ MBL contributes to opsonophagocytosis of C. albicans by neutrophils. ${ }^{10}$ Activation of the complement system by the MBL-MASP complex leads to the opsonization of C. albicans via C3b. ${ }^{10} \mathrm{MBL}$ can also directly opsonize C. albicans by recognition by CR1 on the leukocyte surface. ${ }^{11}$

An association between MBL-deficiency and antiyeast antibodies (ASCA) is observed in Crohn's disease (CD), and this deficiency is frequently associated with a severe phenotype of $\mathrm{CD} .{ }^{12,13}$ Furthermore, CD patients are more frequently colonized by $C$. albicans than control subjects. ${ }^{14}$ Experimental studies show that $C$. albicans aggravates intestinal inflammation induced by dextran sulfate sodium (DSS) in

\footnotetext{
${ }^{1}$ Université Lille Nord de France, Lille, France. ${ }^{2}$ UDSL, Lille, France. ${ }^{3}$ INSERM U995, Lille, France. ${ }^{4}$ CHRU Lille, Lille, France. ${ }^{5}$ Service de Parasitologie Mycologie, Pole de Biologie Pathologie Génétique, Lille, France. ${ }^{6}$ Department of Pediatrics, Massachusetts General Hospital, Harvard Medical School, Boston, Massachusetts, USA and ${ }^{7}$ Department of Gastroenterology, Icahn School of Medicine at Mount Sinai, New York, New York, USA. Correspondence: S Jawhara (samir.jawhara-3@univ-lille2.fr) 


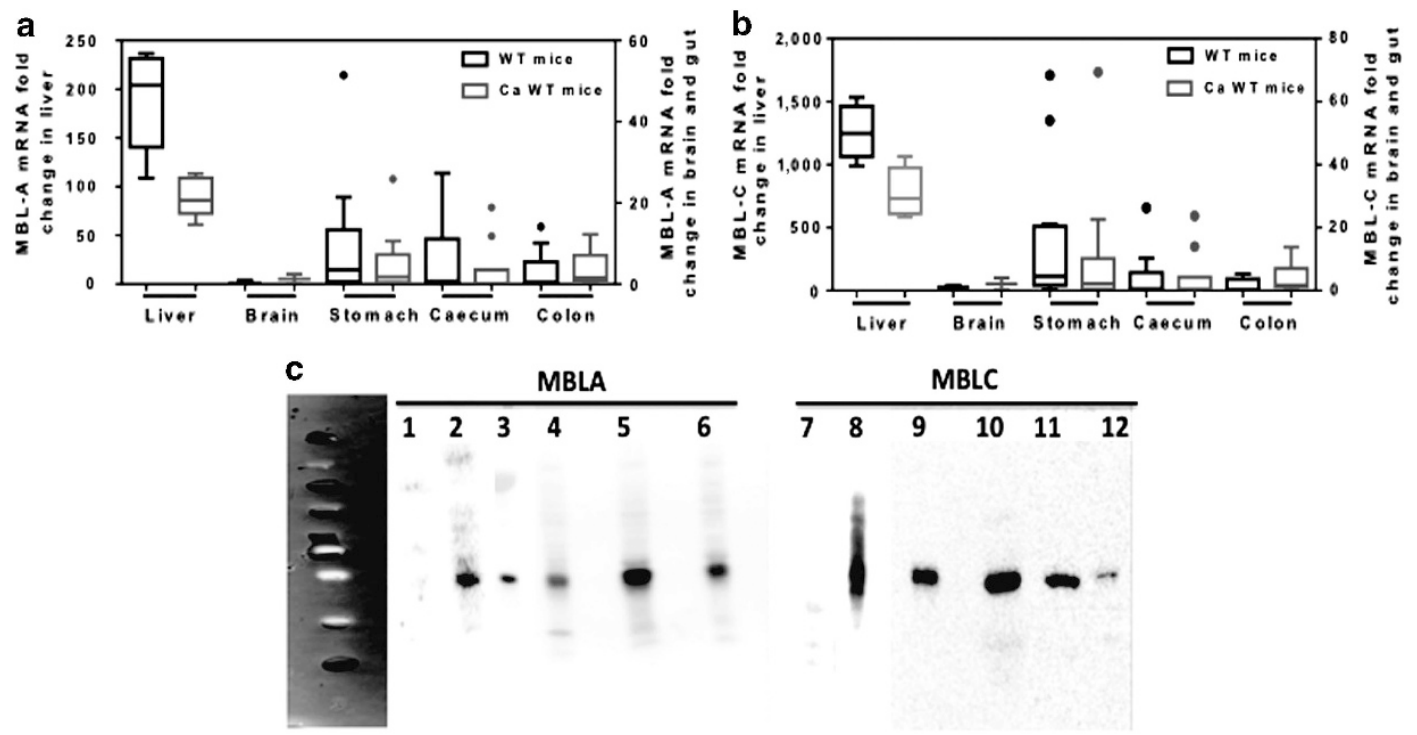

Figure 1 Expression of mannose-binding lectin (MBL)-A and MBL-C in the gastrointestinal tract of wild-type (WT) mice colonized or not colonized with C. albicans. (a, b) mRNA from epithelial cells of the liver (as a positive control), brain (as a negative control), stomach, cecum, and colon was extracted from WT mice colonized or not colonized with $C$. albicans. MBL-A and MBL-C mRNA was detected and amplified using real-time PCR. Results are the mean \pm s.e. of two independent experiments (14 mice per group). (c) Detection of MBL-A and MBL-C proteins in the liver (as a positive control), brain (as a negative control), stomach, cecum, and colon from WT mice colonized or not colonized with $C$. albicans by western blotting. Anti-MBL-A and anti-MBL-C monoclonal antibodies detected a major band of $32 \mathrm{kDa}$ corresponding to the molecular weight of MBL proteins in the liver (lines 2 and 8), stomach (lines 4 and 10), cecum (lines 5 and 11), and colon (lines 6 and 12) from WT mice colonized with C. albicans. Both MBL-A and MBL-C were not detected in the brain (lines 1 and 7). Recombinant mouse MBL-A and MBL-C proteins were used as controls (lines 3 and 9).

mice, and conversely, DSS colitis promotes C. albicans colonization. ${ }^{15}$ Muller et al. ${ }^{16}$ have shown that DSS-induced colitis is exacerbated in MBL-deficient mice infected with $C$. albicans when compared with wild-type (WT) mice. MBL by interaction with Toll-like receptor TLR-2 or TLR-4, inhibits $C$. albicans-induced interleukin (IL)-8 and tumor necrosis factor$\alpha$ production from THP- 1 cells. ${ }^{17}$ The question is whether circulating MBL comes only from the liver and exerts an opsonization effect by eliminating fungal infection/colonization or whether MBL is also produced and released locally in the GI tract itself after fungal challenge. In the present study, we explored the expression of MBL in the gut epithelium and examined its impact on modulation of intestinal inflammation and $C$. albicans elimination.

\section{RESULTS}

Detection of MBL-A and MBL-C mRNA in the GI tract of WT mice colonized or not colonized with $\boldsymbol{C}$. albicans

To examine whether the MBL transcript can be detected in all parts of the gut in response to C. albicans, real-time PCR was performed on the mRNA of epithelial cells from the stomach, cecum, and colon of mice colonized with C. albicans for 7 days (Figure 1). MBL-A and MBL-C mRNA was detected in the epithelial cells of the stomach, cecum, and colon of WT mice colonized with C. albicans and control mice (Figure 1a,b). The expression of MBL-A and MBL-C mRNA was not significantly different in WT mice and WT mice colonized with C. albicans.

Western blotting also confirmed the presence of MBL-A and MBL-C proteins in the stomach and cecum of mice colonized with C. albicans (Figure 1c). Anti-MBL-A and anti-MBL-C monoclonal antibodies labeled a major band of $32 \mathrm{kDa}$ and predicted the molecular weight of MBL proteins. MBL was expressed at a very low level in the colon and was also detected by western blotting. These data suggest that both MBL-A and MBL-C are expressed in the GI tract.

\section{C. albicans colonization increases the expression of MBL-A and MBL-C in the GI tract of mice}

To assess the influence of $C$. albicans colonization on MBL-A and MBL-C expression levels in the gut, WT mice were challenged by gavage with a single inoculum of C. albicans, and the presence of $C$. albicans in the stools was followed for 7 days. High numbers of $C$. albicans colony-forming units (CFU) were recovered from stools on day 7 . This indicates high expression levels of both MBL-A and MBL-C in all parts of the mouse gut colonized with C. albicans when compared with levels in the control mouse gut (Figure 2a,b).

In order to determine the localization of MBL in the GI tract, immunohistofluorescence was performed on stomach sections (Figure 2c). MBL-A and MBL-C expression was detected in epithelial cells of the mouse stomach, and this expression was restricted to the outer mucosal layer of the stomach.

In order to exclude the contribution of MBL from the liver, we employed a tissue culture model (Figure 3). The colons from WT mice were collected, cleaned of food contents with phosphatebuffered saline (PBS), and then incubated with C. albicans combined or not combined with the peroxisome proliferatoractivated receptor- $\gamma$ (PPAR $\gamma$ ) agonist (pioglitazone, $0.1 \mathrm{~mm}$ ) for 

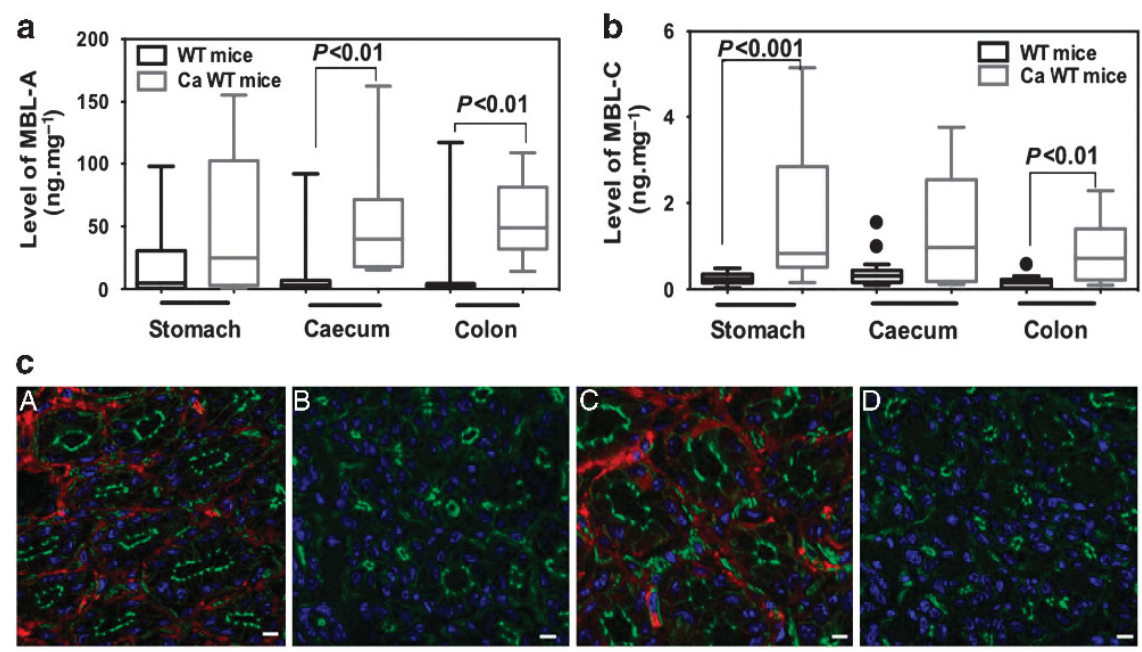

Figure 2 Determination of the expression levels of mannose-binding lectin (MBL)-A and MBL-C in the gastrointestinal tract of wild-type (WT) mice colonized or not colonized with $C$. albicans. $(\mathbf{a}, \mathbf{b})$ After 7 days of $C$. albicans challenge, the expression of MBL-A and MBL-C was determined in homogenates of epithelial cells from the stomach, cecum, and colon using anti-MBL-A and anti-MBL-C monoclonal antibodies. Liver and brain were used as positive and negative controls. Results are the mean \pm s.e. of two independent experiments. (c) Detection by immunofluorescence of MBL-A and MBL$\mathrm{C}$ expression in the stomach of WT mice and MBL-KO (knockout) colonized with $C$. albicans. Immunohistofluorescence of stomach sections from WT mice and MBL-KO mice with (A, B) anti-MBL-A and (C, D) anti-MBL-C monoclonal antibodies (red). The sections were stained with phalloidin F-actin cytoskeleton (green) and counterstained with DAPI (4,6-diamidino-2-phenylindole; blue counterstaining).

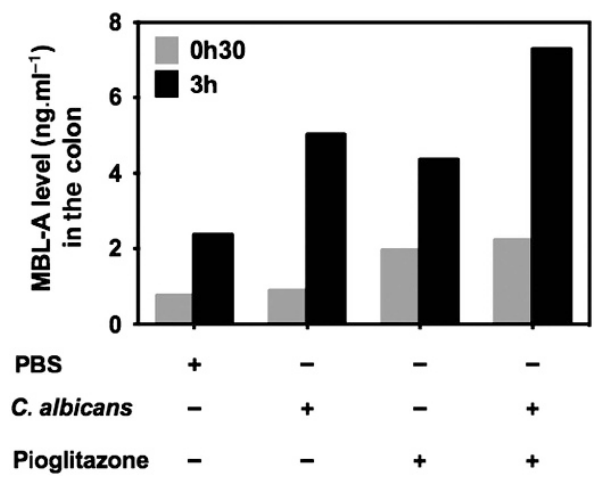

Figure 3 Mouse intestinal explant culture produced mannose-binding lectin (MBL) after $C$. albicans sensing alone or with pioglitazone treatment. The level of MBL-A from colons of wild-type mice increased in the supernatant at $3 \mathrm{~h}$ after $C$. albicans sensing combined or not with pioglitazone. Histograms are representative of two independent experiments involving three mice per group. PBS, phosphate-buffered saline.

$3 \mathrm{~h}$. We observed an increase in the level of MBL in the supernatant in a time-dependent manner.

\section{Opsonization of $C$. albicans by MBL is essential to reduce colonization in mice}

To determine whether MBL, which is produced from the GI tract, is involved in the elimination of C. albicans, mice were challenged orally with $C$. albicans and also received a MBL ligand ( $\alpha$-mannoside residues of Saccharomyces cerevisiae), which is able to block MBL, for 7 days by gavage (Figure 4). Administration of $\alpha$-mannoside residues significantly increased C. albicans colonization in the feces, colon and cecum of WT mice (Figure 4a-c). Furthermore, WT mice treated with $\alpha$-mans were not more intensively colonized with
C. albicans than MBL-KO mice treated or not with $\alpha$-mans (Figure 4). These results suggest that saturation of MBL with $\alpha$ mannosides promotes $C$. albicans colonization in WT mice.

\section{$M B L$ deficiency exacerbates intestinal inflammation and $C$. albicans colonization}

To further assess the involvement of intestinal MBL in $C$. albicans colonization, regulation of homeostasis, and pathogenesis of colitis in the gut, WT mice with a targeted deletion of $M B L-A$ and $M B L-C$ genes were used in the DSS model that mimics human inflammatory bowel disease. MBL-knockout (KO) and WT mice received a single inoculum of C. albicans and were treated with DSS for 2 weeks to induce colitis. Mice were weighed daily and both rectal bleeding and the consistency of stools were examined (Figure 5a). There was no significant difference between MBL-KO and WT mice receiving $C$. albicans alone, in terms of clinical inflammation score (Figure 5a). After DSS treatment, combined with $C$. albicans challenge or not, the clinical score of MBL-KO mice was significantly higher than that of WT mice (with $C$. albicans challenge, the clinical score was increased, but not significantly, in MBL-KO mice). Examination of colonic sections from MBL-KO mice revealed diffuse leukocyte infiltration and epithelial damage when compared with WT mice (Figure 5b).

To assess C. albicans colonization, the amount of yeast in the stools was analyzed daily for 2 weeks. The number of C. albicans $\mathrm{CFU}$ recovered in the stools was significantly higher in MBLKO mice than in WT mice (Figure 6). To evaluate C. albicans colonization in the gut, the number of yeasts adhering to the stomach, cecum, and colon was determined. Significantly higher numbers of CFU were observed in the stomach, cecum, and colon of MBL-KO mice compared with WT mice 

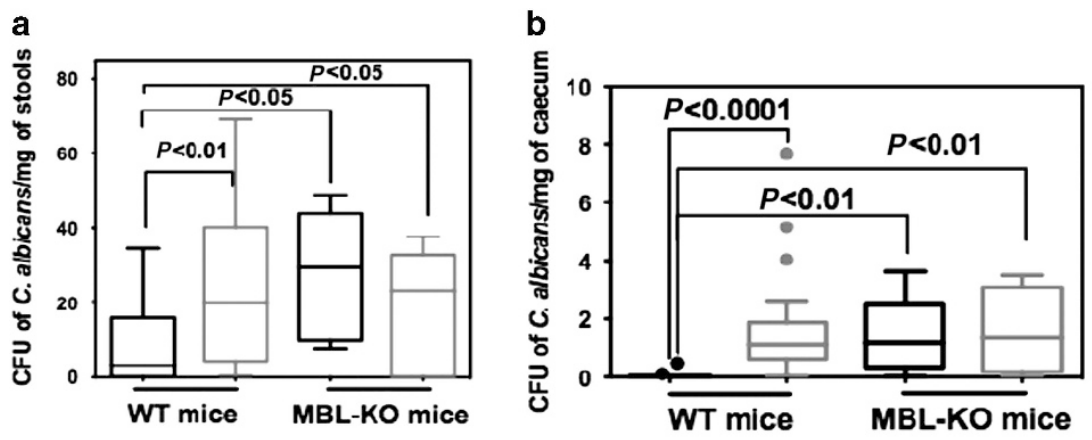

口 C. albicans

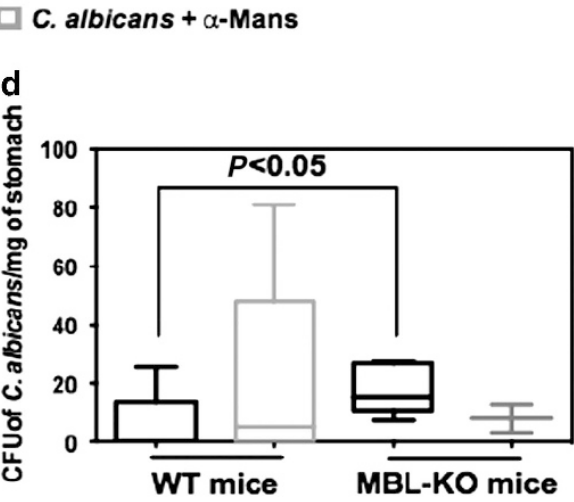

Figure 4 Blocking the carbohydrate recognition domain of mannose-binding lectin (MBL) with $\alpha$-mannosides ( $\alpha$-Mans) increased C. albicans colonization in wild-type (WT) mice. WT mice and MBL-KO (knockout) mice were treated daily for 7 days with $\alpha$-Mans (500 $\mu \mathrm{g}$ per day) to block the carbohydrate recognition domain of MBL in the gut. (a) The number of $C$. albicans colony-forming units (CFU) recovered from stools at day 7 . Results are the mean \pm s.d. of 20 mice per group. ${ }^{*} P<0.05$ for CaWT mice vs. CaWT mice treated with $\alpha$-Mans. (b-d) Number of $C$. albicans CFU recovered from the colon, cecum, and stomach. Results are the mean \pm s.d. of 20 mice per group.

(Figure 6a-c). These results were correlated with immunohistofluorescence, which revealed the presence of large quantities of hyphal and blastoconidial forms of C. albicans either in the lumen or adhering to the stomach epithelium (Figure 6e).

After oral challenge, C. albicans disseminated from the GI tract to the lungs and kidneys of MBL-KO mice, while no $C$. albicans dissemination was observed in WT mice (Figure 7a,b).

\section{MBL modulates the expression of pro-inflammatory cytokines and innate immune receptors in the colon}

To understand the mechanism by which intestinal MBL modulates the inflammatory mediator responses in the pathogenesis of colitis and $C$. albicans colonization, the expression levels of cytokine, including IL-1 $\beta$, IL-6, IL-17, and IL-23, were assessed in colons from MBL-KO and WT mice (Figure 8). IL-1 $\beta$ mRNA and protein expression levels increased significantly in Candida DSS WT mice as compared with DSS WT mice. Furthermore, we found no significant difference in IL- $1 \beta$ mRNA and protein expression levels between MBL-KO DSS and MBL-KO Candida DSS mice (Figure 8a,b). On other the hand, we noted that IL-23 mRNA and protein expression levels were significantly lower in Candida DSS MBL-KO mice than in Candida DSS MBL-KO mice (Figure 8c,d). C. albicans colonization increased the expression of IL-6 mRNA in the colon of WT mice, while the expression for this cytokine decreased in the colons of MBL-KO mice (Figure 8f). In contrast, both IL-17 expression level was significantly increased in MBL-KO mice treated with DSS when compared with WT mice, but C. albicans colonization contributed to downregulation of IL-17 expression in MBLdeficient mice (Figure 8e). To explore whether intestinal MBL cooperates with other receptors in response to C. albicans colonization and colitis, the expression levels of TLR-2, TLR-4, and dectin-1 were examined (Figure 8g-i). With DSS treatment, expression of dectin-1 and TLR-4 was increased, while TLR-2 expression was downregulated in the colons of MBL-KO mice. In response to C. albicans sensing, TLR-4 expression significantly decreased in the colons of MBL-KO CaDSS mice, while expression of dectin-1 was upregulated when compared with that of WT CaDSS mice.

\section{DISCUSSION}

MBL is a soluble pattern recognition molecule, which activates the lectin pathway of the complement system and subsequent inflammatory mechanisms. ${ }^{18,19} \mathrm{MBL}$ deficiency has been associated with a wide spectrum of infectious diseases, including fungal infections. ${ }^{20}$ The biological activity of MBL from the liver has been studied extensively in the blood, in terms of opsonization and activation of the complement system, but few studies have shown the role of MBL in intestinal homeostasis. One of the aims of the present study was to determine 


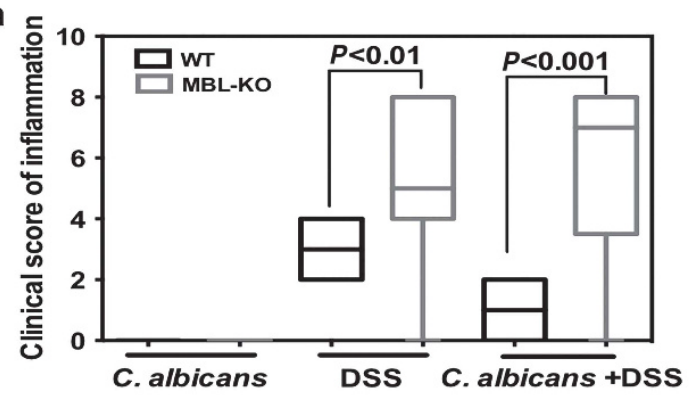

b

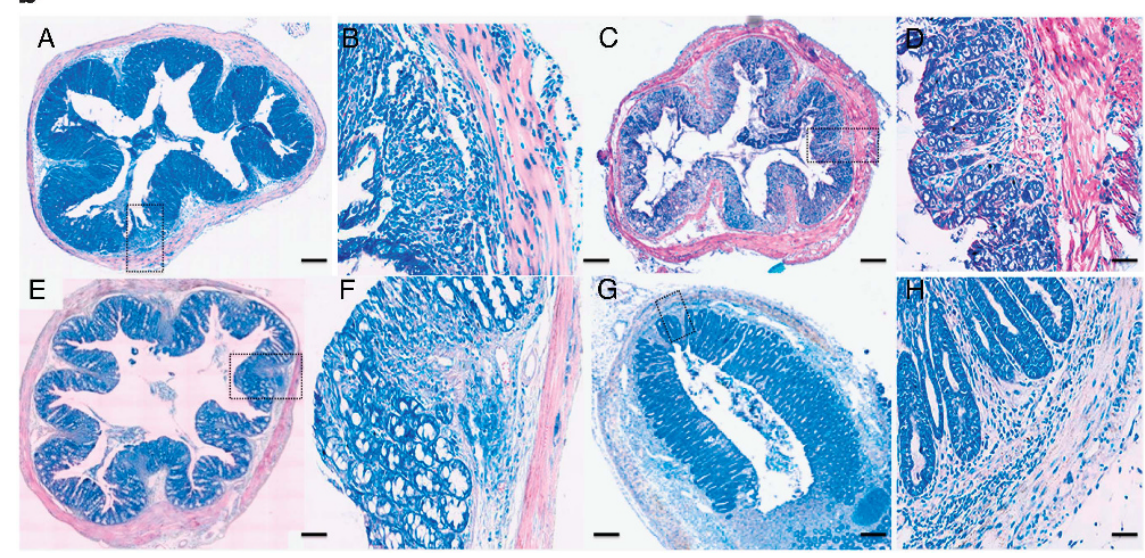

Figure 5 Clinical inflammation score and histological analysis after dextran sodium sulfate (DSS) treatment and C. albicans colonization in mannosebinding lectin knockout (MBL-KO) mice and wild-type (WT) mice. (a) A single inoculum of $10^{7} \mathrm{C}$. albicans was administered to mice on day 1 , and low doses of DSS (1.5\%) were given in the drinking water for 2 weeks. Clinical score was determined by assessing weight loss, change in stool consistency, and the presence of gross bleeding. The clinical score ranged from 0 to 8 . $P<0.01$ for DSS MBL-KO mice vs. DSSWT mice; and $P<0.01$ for $C a n d i d a D S S$ MBL-KO mice vs. Candida DSS WT mice. Results are the mean \pm s.d. of 23 mice per group. (b) Histological analysis of the colon in C. albicans and DSSinduced colitis. Subpanels (A, C) correspond to colon sections from WT-DSS and WT Candida + DSS mice, respectively. Subpanels (E, G) correspond to colon sections from MBL-KO DSS and MBL-KO Candida+DSS mice, respectively. The colon sections from both MBL-KO and WT mice show an inflammatory cell infiltrate in colonic wall structures (subpanels $(\mathbf{D}, \mathbf{H})$ ). The colon sections from Candida + DSS MBL-KO mice exhibit a strong inflammatory cell infiltrate in colonic wall structures and massive tissue destruction (subpanel E). The scale bars represent $50 \mu \mathrm{m}(\mathbf{A}, \mathbf{C}, \mathbf{E}, \mathbf{G})$ and $10 \mu \mathrm{m}$ (B, D, F, H).

whether MBL could be produced locally by gut epithelial cells in response to intestinal inflammation, combined with C. albicans colonization or not, and whether this lectin had a critical role in the intestinal epithelium defense against infection.

In this study, we detected the mRNA transcript and MBL protein throughout the digestive tract. Moreover, colonization with $C$. albicans induced MBL protein production and promoted the elimination of $C$. albicans from the gut. MBL-C was preferentially expressed in the stomach and colon after C. albicans colonization, while MBL-A levels were significantly higher in the cecum and colon of C. albicanscolonized mice when compared with healthy mice.

Besides, the data from mouse intestinal explant culture revealed the role of epithelial cells in the secretion of MBL after C. albicans sensing alone or with pioglitazone treatment, excluding the role of MBL from the liver itself in this in vitro model. Intestinal inflammation induced by DSS did not increase the production of MBL protein when compared with mice colonized with $C$. albicans, indicating that sensing of fungi by epithelial cells increased MBL production. MBL level was independent of DSS-induced colitis. These data are consistent with clinical studies showing that patients with candidemia have high levels of circulating MBL after infection. ${ }^{9}$ Considering that mannans are the principal ligand of MBL and are able to block it, ${ }^{21}$ we found that oral administration of mannans to mice increased C. albicans colonization. Anti-MBL antibodies were then administered and were found to promote $C$. albicans colonization (data not shown). These results correlate with previous studies showing that administration of mannans increased C. albicans colonization in mice. ${ }^{22}$ By confocal microscopy, MBL was found to be produced preferentially in the upper layers of the intestinal mucosa. Collectively, these data show that MBL has an important role in the defense against C. albicans in the gut mucosa during colonization/ infection. This then led us to investigate the impact of MBL deficiency on intestinal homeostasis by employing the DSSinduced colitis model. This DSS model mimics perfectly the human pathogenesis of irritable bowel disease and promotes $C$. albicans colonization in mice. Regardless of intestinal inflammation, C. albicans colonization was much higher in MBL-KO mice than in WT mice, and the stomach was the most colonized part of the digestive tract. ${ }^{23}$ In contrast to WT mice, in whom no dissemination of C. albicans could be observed, DSS-induced 

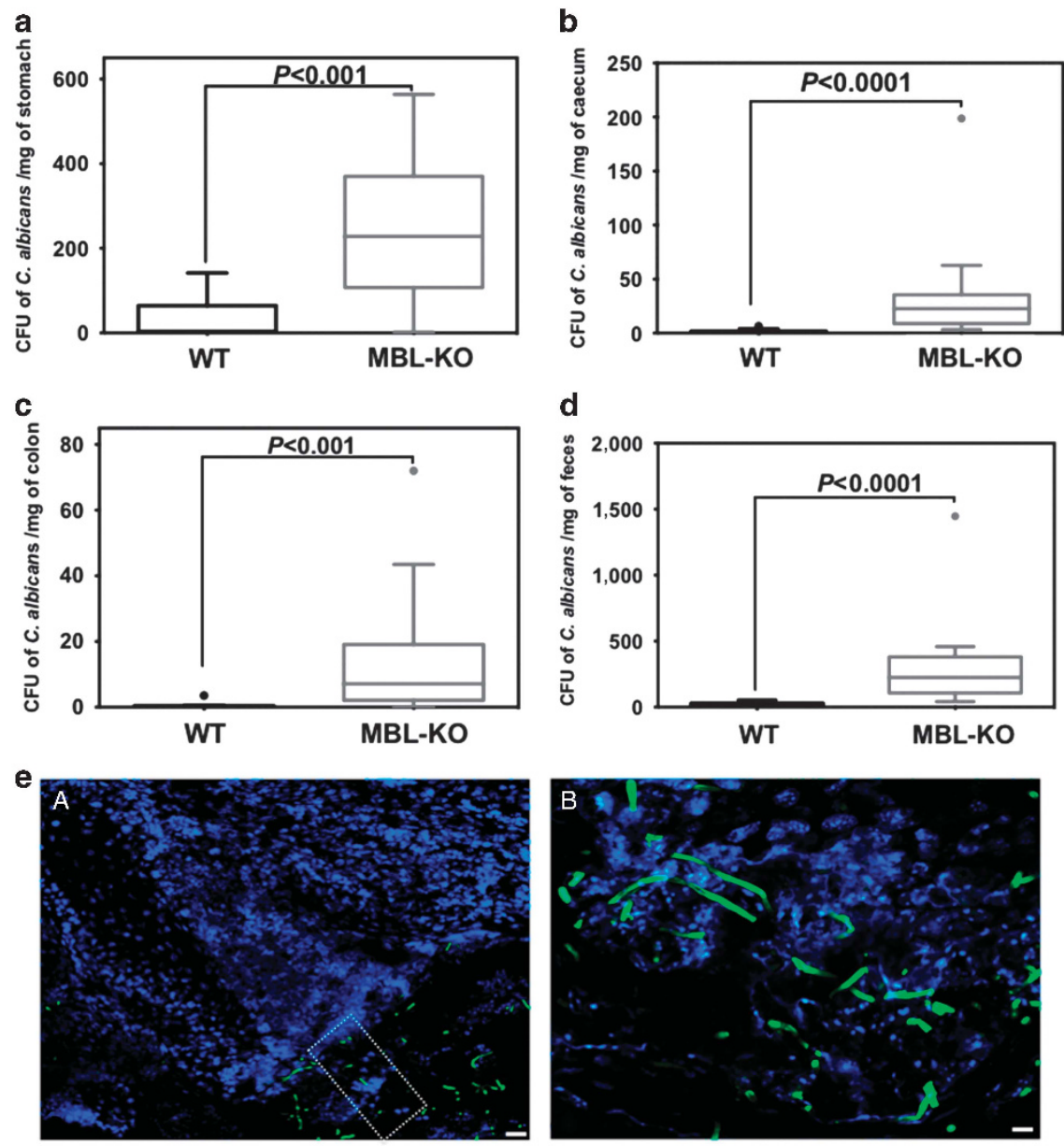

Figure 6 Mannose-binding lectin (MBL) deficiency promoted $C$. albicans colonization in mice. (a-d) The number of $C$. albicans colony-forming units (CFU) recovered from the stomach, cecum, colon, and stools in MBL-KO (knockout) and wild-type (WT) mice colonized with C. albicans for 14 days. Data are the mean \pm s.d. of 23 mice per group. $P<0.001$ for Candida + DSS MBL-KO mice vs. Candida+DSS WT mice. (e) Immunohistofluorescence staining of stomach section from Candida + DSS MBL-KO mouse. C. albicans hyphal forms can be observed in the stomach lumen and adhering to the epithelial cells. The section was counterstained with DAPI (4,6-diamidino-2-phenylindole; blue counterstaining). The scale bars represent $50 \mu \mathrm{m}(\mathrm{A})$ and $5 \mu \mathrm{m}$ (B).
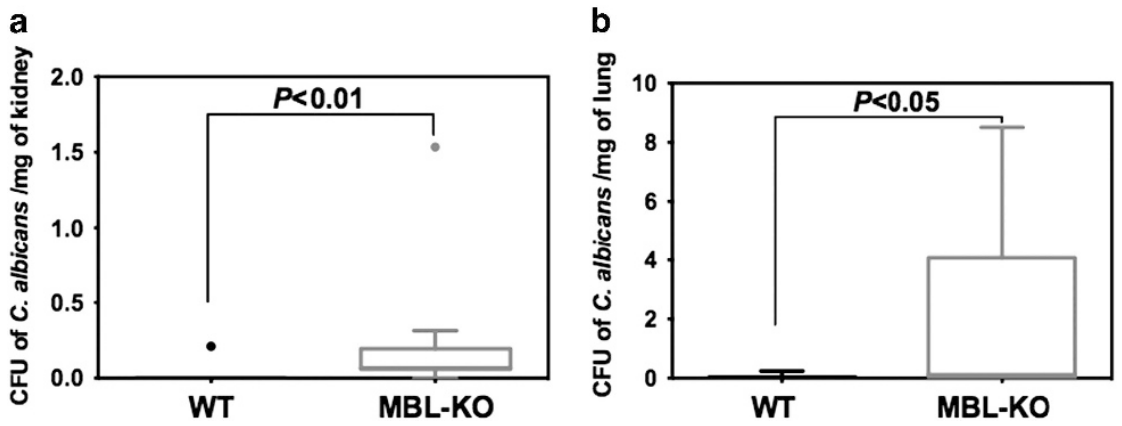

Figure 7 The lack of mannose-binding lectin (MBL) promoted $C$. albicans dissemination into the kidneys and lungs. (a, b) After 14 days of $C$. albicans challenge and dextran sodium sulfate (DSS) treatment, the kidneys and lungs were harvested and homogenized in phosphate-buffered saline, and serial dilutions of the homogenates were plated onto Sabouraud's agar for quantification of $C$. albicans colony-forming units (CFU). Data are the mean $\pm s$.d. of 23 mice per group. (a) $P<0.01$ for Candida + DSS MBL-KO mice vs. Candida + DSS WT mice. (b) $P<0.05$ for Candida + DSS MBL-KO mice vs. Candida + DSS WT mice.

colitis increased the fungal burden in the kidneys and lungs of MBL-KO mice. These observations provide in vivo evidence that MBL contributes to innate immune protection in the stomach, lungs, and kidneys and are similar to those of another study that showed an increase in fungal burden in the kidney and lungs of MBL-KO mice. ${ }^{16}$ In the mouse model of IC, MBL deficiency was strongly associated with increased C. albicans infections. $^{20}$ 

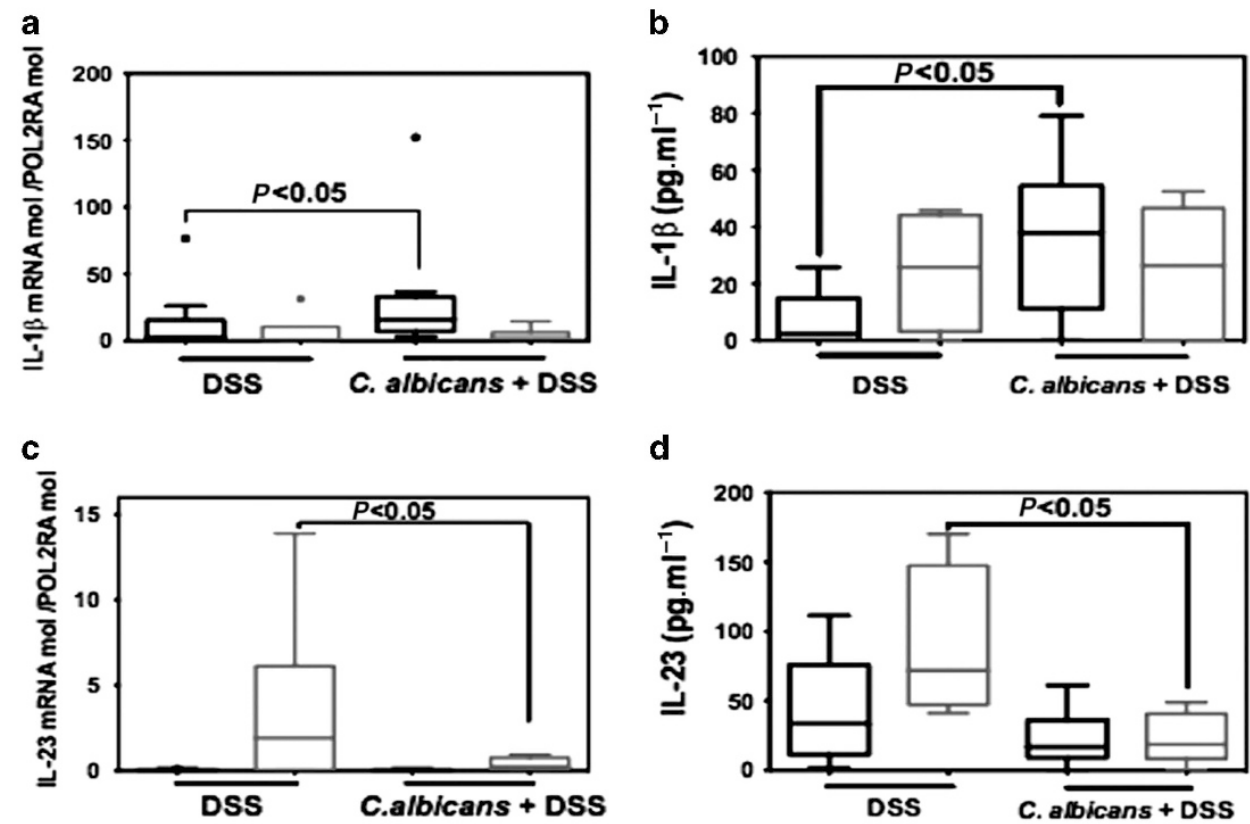

d
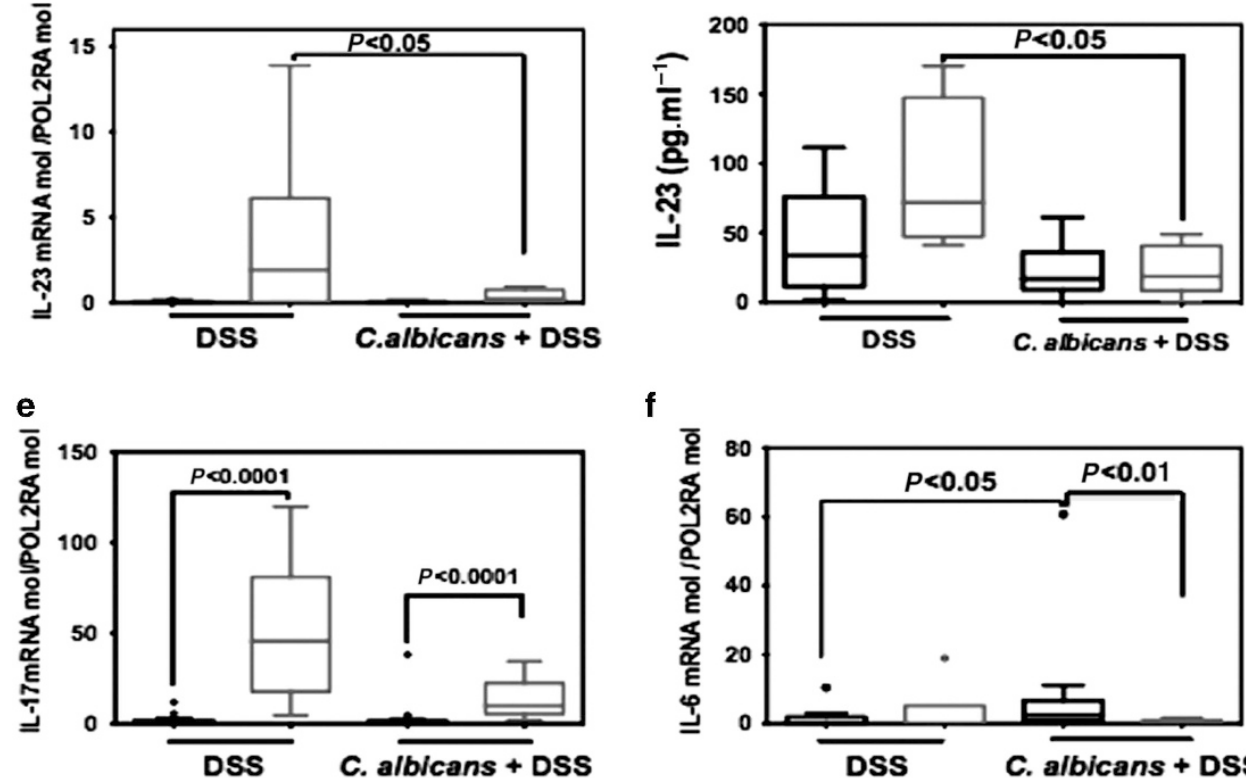

f
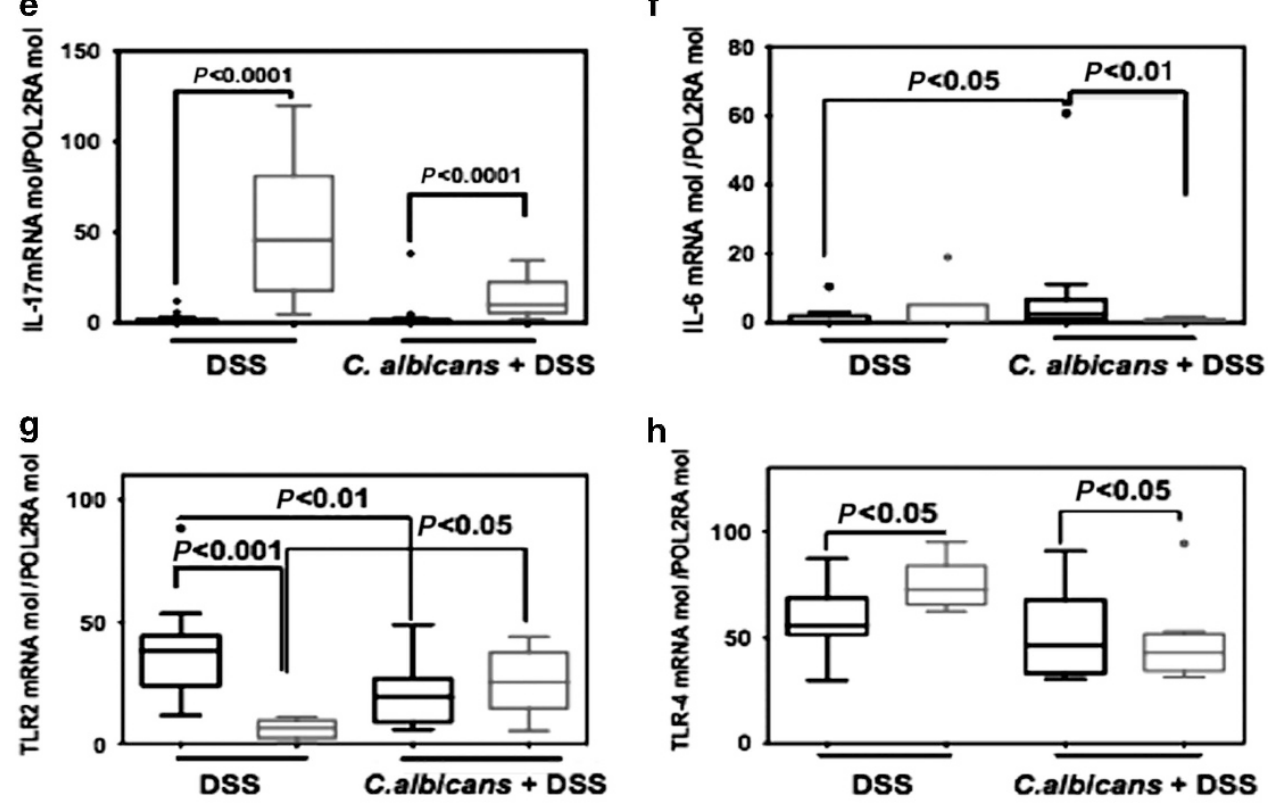

h
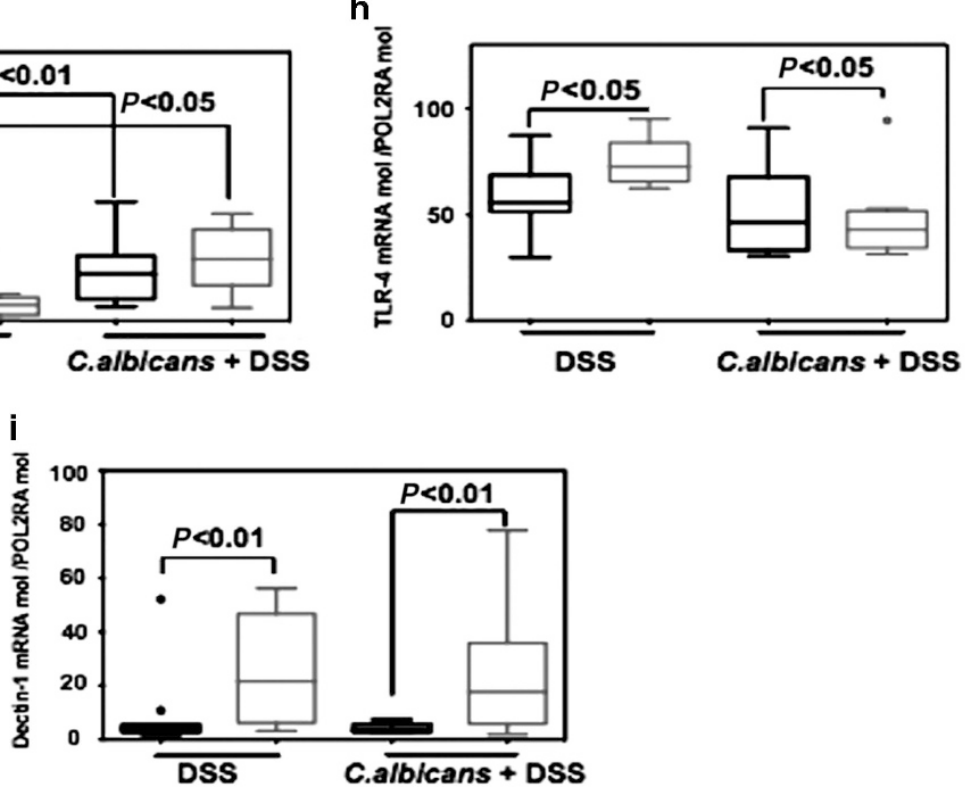

Figure 8 Mannose-binding lectin (MBL) deficiency impacts on the expression of pro-inflammatory cytokines and innate immune receptors in the colon. (a-d) mRNA and protein expression levels of interleukin (IL)-1 $\beta$, and IL-23 in mouse colons. (e-i) Relative expression levels of IL-17, II-6, Toll-like receptor (TLR)-2, TLR-4, and Dectin-1 mRNA in mouse colons. Data are the mean \pm s.e. of 23 mice per group. DSS, dextran sodium sulfate. 
MBL can also modulate inflammatory mediators, in particular IL-1 $\beta$ and IL-6, which have an important role in the development of intestinal inflammation and show a dramatic increase in the colonic mucosa during disease. ${ }^{24,25}$ We observed that colonization by $C$. albicans promoted the expression of both IL- $1 \beta$ and IL- 6 in the colon of DSS WT mice but not in DSS MBL-KO mice. These data are similar to those of other studies showing that MBL modulates the release of both IL- $1 \beta$ and IL- 6 production from monocytes. ${ }^{26,27}$ In contrast, IL-17 and IL-23 expression was significantly higher in MBLKO mice treated with DSS when compared with WT mice. These data suggest that MBL prevents an excessive inflammatory response and can possibly act as a mannose receptor or dendritic cell-specific intercellular adhesion molecule-3-grabbing non-integrin that inhibits $\mathrm{T}$ helper type 17 (Th17) and increases Th1 production. ${ }^{28}$ In several previous studies, the increased expression of IL23 and IL17 was shown to be pivotal in the development of chronic mucosal inflammation and to promote intraepithelial lymphocyte inflammatory responses and cytotoxicity. ${ }^{29,30}$ Many of the inflammatory consequences of C. albicans-host cell interaction are mediated through pattern-recognition receptors, including TLRs, dectin-1, and MBL. $^{31,32}$ We showed that MBL deficiency altered the expression of TLR-4 but not TLR-2 in the colons from mice receiving DSS and challenged with $C$. albicans. These data suggest that intestinal MBL is likely cooperating with TLR-4 to amplify the antifungal response. Laisk et al. ${ }^{33}$ showed that mutations in both TLR- 4 and MBL2 have a role in receptiveness to pathogens causing genital tract infections. Besides, we observed that deficiency of MBL leads to an increase in the expression level of Dectin-1 in CaDSS mice, promoting Th17 response. It has been reported that Dectin-1 activation via fungal infection induces the production of IL-17 and IL-23 from dendritic cells. ${ }^{34,35}$ Additionally, we observed that, in response to C. albicans sensing, TLR-2 expression increased significantly in the colons of MBL-KO mice, suggesting that this increased level could likely be related to a compensatory response to MBL deficiency, attempting to enhance the antifungal host defense. Mechanistically, PPAR $\gamma$ activation that leads to MBL production in the epithelial cells is possibly involved in the regulation of TLR signaling. Appel et al. ${ }^{36}$ showed that PPAR $\gamma$ agonists inhibit TLR-mediated dendritic cells via mitogen-activated protein kinase and nuclear factor- $\kappa \mathrm{B}$ pathways. It is worth noting that TLR-2 alone can sense phospholipomannans of $C$. albicans or cooperate with other receptors such as dectin-1 ( $\beta$-glucans) or galectin-3 ( $\beta-1,3$ mans) to induce a pro-inflammatory response. $^{37-40}$

In conclusion, this study shows that MBL is expressed in gut epithelial cells in response to C. albicans sensing. Mice deficient in MBL are highly susceptible to C. albicans colonization. This lectin is pivotal in intestinal inflammation and host defense by modulating the inflammatory mediator responses (Th1/Th17) and amplifying the expression of certain receptors (dectin-1 and TLR-4). Although Muller et al. ${ }^{16}$ showed that MBL expression was virtually undetectable in the intestinal mucosa from healthy subjects or patients with $\mathrm{CD}$, our study using Caco-2 human colorectal cells stimulated with different PPAR $\gamma$ agonists (amino-phenyl-methoxy-propionic acid, 5-aminosalycilate, or pioglitazone) showed a high expression of MBL-2 mRNA by microarray and quantitative reverse transcriptasePCR analyses (see Supplementary Data online). Of note, $\operatorname{PPAR} \gamma$ contributes to increased recognition and phagocytosis of unopsonized C. albicans and PPAR $\gamma$ activation reduces $C$. albicans colonization in the murine digestive tract. ${ }^{41,42}$ These data reveal that MBL-2 expression is regulated by PPAR $\gamma$ in the epithelial cells. Additionally, we analyzed the expression of epithelial MBL in two human non-inflamed colons collected from $C D$ patients. Our preliminary results show that these samples produced MBL after C. albicans or C. albicans + $\operatorname{PPAR} \gamma$ agonist incubation (pioglitazone). These first pilot experiments obtained from human tissue culture are similar to those produced by mouse tissue culture (see Supplementary Data). It is tempting to assess the expression of epithelial MBL in human gut biopsies from patients with IC or patients with multiple sites of $C$. albicans colonization. Finally, this present study provides insights into the physiological role of MBL in the digestive tract in the mediation of the epithelial cell defense against pathogens and maintenance of intestinal homeostasis.

\section{METHODS}

Animals. MBL-KO mice (MBL-A and MBL-C double knockout) were provided by Dr K Takahashi (Department of Pediatrics, Massachusetts General Hospital, Harvard Medical School, Boston, MA). Both WT $(\mathrm{C} 57 \mathrm{BL} / 6)$ mice and MBL-KO mice were bred at Charles River Laboratories (Saint-Germain-sur-l'Arbresle, France). Eight-to-10week-old female mice were used in this study. The mice were maintained in a 12-h alternating light/dark cycle and supplied with food and water ad libitum. Two sets of experiments were performed independently. Mouse survival and body weight were monitored daily for 14 days. All efforts were made to minimize animal suffering. Mice with body weight loss of $>20 \%$ initial body weight were euthanized humanely by inhalation of $5 \%$ isoflurane followed by cervical dislocation.

Ethics statement. Experiments were performed according to the protocols approved by the subcommittee on Research Animal Care of the Regional Hospital Centre of Lille, France and in accordance with European legal and institutional guidelines (86/609/CEE) for the care and use of laboratory animals.

Yeast strain. C. albicans Sc5314 strain was used throughout this study. ${ }^{43}$ C. albicans isolates were grown in Sabouraud's dextrose broth at $37^{\circ} \mathrm{C}$ in a shaking incubator for $18 \mathrm{~h}$.

Inoculum preparation and induction of colitis. WT mice were administered $10^{7}$ C. albicans cells in $500 \mu \mathrm{l}$ PBS by gavage. Feces were collected daily from each mouse to follow up C. albicans colonization. To block the MBL carbohydrate recognition domain in the gut by $\alpha$ mannosides, WT mice and MBL-KO mice were administered $10^{7} \mathrm{C}$. albicans cells in $500 \mu \mathrm{l}$ PBS and given $500 \mu \mathrm{g}$ mannan in $500 \mu \mathrm{l}$ PBS (Sigma-Aldrich, Saint Quentin Fallavier, France) daily for 7 days. For DSS-induced colitis, both WT mice and MBL-KO mice received $1.5 \%$ DSS (MW 36-50 kDa; MP Biomedicals, Illkirch, France) in drinking water from day 1 to day 14 to induce colitis. At day 0 , mice were administered $10^{7}$ C. albicans cells in $500 \mu$ PBS by gavage. The clinical inflammation score was determined daily based on weight loss, stool consistency, and the presence of blood in the stools. The clinical scores ranging from 0 to 8 were calculated as described elsewhere. ${ }^{15,44}$ The 
presence of yeast in the GI tract was assessed daily by performing plate counts from feces (approximately $0.1 \mathrm{~g}$ per sample). ${ }^{15}$ Feces were collected daily from each mouse to follow up C. albicans colonization. Feces were suspended in $1 \mathrm{ml}$ PBS and ground in a glass tissue homogenizer. Serial dilutions of the homogenates were plated on Sabouraud's agar. The colonies were counted after $48 \mathrm{~h}$ incubation at $37^{\circ} \mathrm{C}$. The results were noted as CFU mg ${ }^{-1}$ feces. Mice were killed at day 14 by cervical dislocation after anesthesia with $5 \%$ isoflurane. To determine C. albicans colonization in the gut, the GI tract was removed from killed mice, and the stomach, cecum, and colon were separated and analyzed. The tissues were cut longitudinally. After removal of the intestinal contents, the tissues were washed several times with PBS to minimize surface contamination from organisms present in the lumen. To assess C. albicans dissemination into the lungs and kidneys, organs were harvested, weighed, and homogenized in $1 \mathrm{ml}$ PBS with an UltraTurax homogenizer (IKA, Staufen, Germany). Serial dilutions of the homogenates were plated on Sabouraud's agar. The results were noted as $\mathrm{CFU} \mathrm{mg} \mathrm{mg}^{-1}$ of organ.

Histological score. Rings of the transverse part of the colon were fixed overnight in $4 \%$ paraformaldehyde acid and embedded in paraffin, and histological analysis was performed by staining the cross-sections ( $4 \mu \mathrm{m}$ thick) with hematoxylin-eosin (Sigma-Aldrich). Histological scores were evaluated by two independent blinded investigators who observed two sections per mouse at magnifications of $\times 10$ and $\times 100 .{ }^{15}$ The histological score ranged from 0 (no changes) to 6 (extensive cell infiltration and tissue damage).

Immunohistofluorescence analysis. After killing, tissues were incubated in $4 \%$ paraformaldehyde overnight at $4{ }^{\circ} \mathrm{C}$ and then embedded in OCT compound (Tissue-tek, Sakura Finetek, Torrance, CA). Slides were rehydrated with TBS (Tris-buffered saline) containing $0.05 \%$ Tween (TBS/Tw $0.05 \%$ ) and blocked with $1 \%$ bovine serum albumin (Sigma-Aldrich). For the detection of MBL-A and MBL-C in tissue, slides were incubated with anti-MBL-A and anti-MBL-C rat antibodies (Cedarlane, Burlington, Ontario, Canada) overnight at $4{ }^{\circ} \mathrm{C}$. After several washes with TBS/Tw (0.05\%), slides were incubated with goat anti-rat IgG coupled with Alexa 568 (Invitrogen, Carlsbad, CA). Epithelial cells were stained with Alexa 488 phalloidin (Life Technologies, Carlsbad, CA). For the detection of C. albicans in the stomach, slides were incubated with monoclonal antibody 5B2, which reacts with $\beta$-linked oligomannose epitopes of the $C$. albicans cell wall. ${ }^{45}$ The sections were then incubated with fluorescein isothiocyanate conjugated goat anti-mouse IgM (Zymed Laboratories, San Francisco, CA) for $60 \mathrm{~min}$ at $37^{\circ} \mathrm{C}$. The sections were washed with TBS, counterstained with DAPI (4,6-diamidino-2-phenylindole; Vector, Burlingame, CA), and examined by confocal microscopy (Zeiss LSM710, diode $561 \mathrm{~nm}$ DPSS, Iena, Germany).

Determination of MBL-A and MBL-C levels in the organs. To assess the concentration of MBL in the stomach, cecum, and colon of WT mice, a 96-well plate (Nunc-Immuno Maxisorp, Roskilde, Denmark) was coated with $100 \mu \mathrm{l}$ per well mannans of $S$. cerevisiae $\left(1 \mathrm{mg} \mathrm{ml}^{-1}\right)$. After several washes, $100 \mu \mathrm{l}$ of homogenate sample was mixed with $100 \mu \mathrm{l}$ TBS containing calcium and then added to each well for $1 \mathrm{~h}$ at $4{ }^{\circ} \mathrm{C}$. After several washes, mouse monoclonal anti-MBL-C or antiMBL-A antibodies (Cedarlane) were added to each well. After several washes, horseradish peroxidase-conjugated rat anti-mouse IgG (Zymed Laboratories) was added to the plate and incubated at $37^{\circ} \mathrm{C}$ for $30 \mathrm{~min}$. Absorbance was read at $450 \mathrm{~nm}$ (reference filter, $620 \mathrm{~nm}$ ) in a microplate reader (Bio-Rad Laboratories, Hercules, CA). A standard curve was prepared using serial dilutions of recombinant MBL-A and MBL-C proteins (R\&D Systems, Minneapolis, MN).

Real-time mRNA quantification of pro-inflammatory cytokines and innate immune receptors. Total RNA was isolated from the liver, brain, stomach, cecum, and colon using a commercial kit (Nucleospin RNA, Macherey-Nagel, Duren, Germany). RNA quantification was performed by spectrophotometry (Nanodrop; Nyxor Biotech, Paris, France). Reverse transcription of mRNA was carried out in a final volume of $20 \mu \mathrm{l}$ from $1 \mu \mathrm{g}$ total RNA (high-capacity cDNA RT Kit; Applied Biosystems, Carlsbad, CA). cDNA was amplified by PCR using Fast SYBR green (Applied Biosystems) in the one-step system (Applied Biosystems). SYBR green dye intensity was analyzed using the one-step software. All results were normalized to the reference gene, POLR2A. ${ }^{46}$

Quantification of cytokine proteins by enzyme-linked immunosorbent assay. Pro-inflammatory cytokine concentrations (IL-1 $\beta$ and IL-23) from the colons were measured using a commercial enzymelinked immunosorbent assay kit according to the manufacturer's instructions (eBioscience, San Diego, CA). The data are expressed as $\mathrm{pg} \mathrm{ml}^{-1}$.

Western blotting analysis of MBL-A and MBL-C in the gut. Total protein extracts were prepared from the liver, brain, stomach, cecum, and colon homogenates. The proteins were heated for $5 \mathrm{~min}$ in $\beta$-mercaptoethanol-sodium dodecyl sulfate. A ready-to-use sodium dodecyl sulfate-polyacrylamide gel electrophoresis gel (4-20\%; GE Healthcare, Kouterveldstraat, Belgium) was used, according to the manufacturer's instructions. The proteins were then transferred to a nitrocellulose membrane (Protan; Whatman, Kouterveldstraat, Belgium). The nitrocellulose membrane was incubated at room temperature with $5 \%$ bovine serum albumin in $2 \% \mathrm{TBS} / \mathrm{Tw}$. After three washes with $2 \% \mathrm{TBS} / \mathrm{Tw}$, the nitrocellulose membrane was incubated with mouse monoclonal anti-MBL-C or anti-MBL-A antibodies (Cedarlane) at a dilution of $1 / 1,000$ in TBS/Tw2\%, overnight at $4{ }^{\circ} \mathrm{C}$. After five washes in $2 \% \mathrm{TBS} / \mathrm{Tw}$, the nitrocellulose membrane was incubated with a secondary anti-rat IgG antibody coupled to peroxidase (Jackson, Sacramento, CA) at a dilution of $1 / 10,000$ in $2 \%$ $\mathrm{TBS} / \mathrm{Tw}$ for $2 \mathrm{~h}$ at room temperature. The reaction was revealed by chemiluminescence with an ECL Kit (Thermoscientific, Super signal west pico, Waltham, MA), according to the manufacturer's instructions.

Tissue culture in vitro. Colons from WT mice were washed several times with PBS and then incubated with $10^{7}$ C. albicans combined or not combined with pioglitazone $(0.1 \mathrm{~mm})$ in Dulbecco's modified Eagle's medium media containing $1 \%$ penicillin/streptomycin. These tissue samples were incubated at $37^{\circ} \mathrm{C}$ for $4 \mathrm{~h}$. Culture supernatant was collected at $30 \mathrm{~min}$ and $4 \mathrm{~h}$, and detection of MBL-A and MBL-C was performed by enzyme-linked immunosorbent assay, as described above.

Statistical analysis. All statistical analyses were performed with Prism 4.0 from GraphPad (La Jolla, CA) and XLSTAT (Paris, France). Data were analyzed using the Mann-Whitney $U$-test to compare pairs of groups. The results are expressed as the mean \pm s.d. of individual experimental groups. Differences were considered significant when the $P$-value was indicated as follows: $P<0.05 ; P<0.01$; $P<0.001$.

SUPPLEMENTARY MATERIAL is linked to the online version of the paper at http://www.nature.com/mi

\section{ACKNOWLEDGMENTS}

This work was funded by the FP7 Health 260338 "ALLFUN" project "Fungi in the setting of inflammation, allergy and auto-immune diseases: translating basic science into clinical practices". This work was also funded through BIOASTER by AVIESAN (France's National Alliance for Life Sciences and Health grant no. ARN-10-AIRT-03). The authors thank the digestScience Foundation for their support.

\section{DISCLOSURE}

The authors declared no conflict of interest. 


\section{REFERENCES}

1. Jensenius, H., Klein, D.C., van Hecke, M., Oosterkamp, T.H., Schmidt, T. \& Jensenius, J.C. Mannan-binding lectin: structure, oligomerization, and flexibility studied by atomic force microscopy. J. Mol. Biol. 391, 246-259 (2009).

2. Thiel, S. et al. Mannan-binding lectin (MBL)-associated serine protease-1 (MASP-1), a serine protease associated with humoral pattern-recognition molecules: normal and acute-phase levels in serum and stoichiometry of lectin pathway components. Clin. Exp. Immunol. 169, 38-48 (2012).

3. Degn, S.E. et al. Mannan-binding lectin-associated serine protease (MASP)- 1 is crucial for lectin pathway activation in human serum, whereas neither MASP-1 nor MASP-3 is required for alternative pathway function. J. Immunol. 189, 3957-3969 (2012).

4. Hansen, S., Thiel, S., Willis, A., Holmskov, U. \& Jensenius, J.C. Purification and characterization of two mannan-binding lectins from mouse serum. J. Immunol. 164, 2610-2618 (2000).

5. Lionakis, M.S. \& Netea, M.G. Candida and host determinants of susceptibility to invasive candidiasis. PLoS Pathog. 9, e1003079 (2013).

6. Gow, N.A., van de Veerdonk, F.L., Brown, A.J. \& Netea, M.G. Candida albicans morphogenesis and host defence: discriminating invasion from colonization. Nat. Rev. Microbiol. 10, 112-122 (2012).

7. Poulain, D. Candida albicans, plasticity and pathogenesis. Crit. Rev. Microbiol. 2013)

8. Miranda, L.N. et al. Candida colonisation as a source for candidaemia. J. Hosp. Infect. 72, 9-16 (2009).

9. Damiens, S. et al. Mannose-binding lectin levels and variation during invasive candidiasis. J. Clin. Immunol. 32, 1317-1323 (2012).

10. Brouwer, N., Dolman, K.M., van Houdt, M., Sta, M., Roos, D. \& Kuijpers, T.W. Mannose-binding lectin (MBL) facilitates opsonophagocytosis of yeasts but not of bacteria despite MBL binding. J. Immunol. 180, 41244132 (2008).

11. Ghiran, I., Barbashov, S.F., Klickstein, L.B., Tas, S.W., Jensenius, J.C. \& Nicholson-Weller, A. Complement receptor 1/CD35 is a receptor for mannan-binding lectin. J. Exp. Med. 192, 1797-1808 (2000).

12. Bak-Romaniszyn, L. et al. Mannan-binding lectin deficiency in pediatric patients with inflammatory bowel disease. Scand. J. Gastroenterol. 46, 1275-1278 (2011).

13. Schoepfer, A.M. et al. Low Mannan-binding lectin serum levels are associated with complicated Crohn's disease and reactivity to oligomannan (ASCA). Am. J. Gastroenterol. 104, 2508-2516 (2009).

14. Standaert-Vitse, A. et al. Candida albicans colonization and ASCA in familial Crohn's disease. Am. J. Gastroenterol. 104, 1745-1753 (2009).

15. Jawhara, S. et al. Colonization of mice by Candida albicans is promoted by chemically induced colitis and augments inflammatory responses through galectin-3. J. Infect. Dis. 197, 972-980 (2008).

16. Muller, S. et al. Mannan-binding lectin deficiency results in unusual antibody production and excessive experimental colitis in response to mannose-expressing mild gut pathogens. Gut 59, 1493-1500 (2010).

17. Wang, M. et al. Mannan-binding lectin inhibits Candida albicans-induced cellular responses in PMA-activated THP-1 cells through Toll-like receptor 2 and Toll-like receptor 4. PLoS One 8, e83517 (2013).

18. Thiel, S., Frederiksen, P.D. \& Jensenius, J.C. Clinical manifestations of mannan-binding lectin deficiency. Mol. Immunol. 43, 86-96 (2006).

19. Takahashi, K. Mannose-binding lectin and the balance between immune protection and complication. Expert Rev. Antiinfect. Ther. 9, 1179-1190 (2011).

20. Held, K., Thiel, S., Loos, M. \& Petry, F. Increased susceptibility of complement factor B/C2 double knockout mice and mannan-binding lectin knockout mice to systemic infection with Candida albicans. Mol. Immunol. 45, 3934-3941 (2008).

21. Ip, W.K., Takahashi, K., Moore, K.J., Stuart, L.M. \& Ezekowitz, R.A. Mannose-binding lectin enhances Toll-like receptors 2 and 6 signaling from the phagosome. J. Exp. Med. 205, 169-181 (2008).

22. Jawhara, S. et al. Modulation of intestinal inflammation by yeasts and cell wall extracts: strain dependence and unexpected anti-inflammatory role of glucan fractions. PLoS One 7, e40648 (2012).

23. de Repentigny, L., Phaneuf, M. \& Mathieu, L.G. Gastrointestinal colonization and systemic dissemination by Candida albicans and Candida tropicalis in intact and immunocompromised mice. Infect. Immun. 60, 4907-4914 (1992).
24. Coccia, M. et al. IL-1beta mediates chronic intestinal inflammation by promoting the accumulation of IL-17A secreting innate lymphoid cells and CD4(+) Th17 cells. J. Exp. Med. 209, 1595-1609 (2012).

25. Mudter, J. \& Neurath, M.F. II-6 signaling in inflammatory bowel disease: pathophysiological role and clinical relevance. Inflamm. Bowel. Dis. 13, 1016-1023 (2007).

26. Sprong, T. et al. Mannose binding lectin enhances IL-1beta and IL-10 induction by non-lipopolysaccharide (LPS) components of Neisseria meningitidis. Cytokine 28, 59-66 (2004).

27. Jack, D.L., Read, R.C., Tenner, A.J., Frosch, M., Turner, M.W. \& Klein, N.J. Mannose-binding lectin regulates the inflammatory response of human professional phagocytes to Neisseria meningitidis serogroup B. J. Infect. Dis. 184, 1152-1162 (2001).

28. Zenaro, E., Donini, M. \& Dusi, S. Induction of Th1/Th17 immune response by Mycobacterium tuberculosis: role of dectin-1, Mannose Receptor, and DC-SIGN. J. Leukoc. Biol. 86, 1393-1401 (2009).

29. Liu, Z. et al. The increased expression of IL-23 in inflammatory bowel disease promotes intraepithelial and lamina propria lymphocyte inflammatory responses and cytotoxicity. J. Leukoc. Biol. 89, 597-606 (2011).

30. Siakavellas, S.I. \& Bamias, G. Role of the IL-23/IL-17 axis in Crohn's disease. Discov. Med. 14, 253-262 (2012).

31. Iliev, I.D. et al. Interactions between commensal fungi and the C-type lectin receptor Dectin-1 influence colitis. Science 336, 1314-1317 (2012).

32. Netea, M.G., Van Der Graaf, C.A., Vonk, A.G., Verschueren, I., Van Der Meer, J.W. \& Kullberg, B.J. The role of toll-like receptor (TLR) 2 and TLR4 in the host defense against disseminated candidiasis. J. Infect. Dis. 185, 1483-1489 (2002).

33. Laisk, T., Peters, M., Saare, M., Haller-Kikkatalo, K., Karro, H. \& Salumets, A. Association of CCR5, TLR2, TLR4 and MBL genetic variations with genital tract infections and tubal factor infertility. J. Reprod. Immunol. 87, 74-81 (2010).

34. Osorio, F. et al. DC activated via dectin-1 convert Treg into IL-17 producers. Eur. J. Immunol. 38, 3274-3281 (2008).

35. LeibundGut-Landmann, S. et al. Syk- and CARD9-dependent coupling of innate immunity to the induction of $\mathrm{T}$ helper cells that produce interleukin 17. Nat. Immunol. 8, 630-638 (2007).

36. Appel, S., Mirakaj, V., Bringmann, A., Weck, M.M., Grunebach, F. \& Brossart, P. PPAR-gamma agonists inhibit toll-like receptor-mediated activation of dendritic cells via the MAP kinase and NF-kappaB pathways. Blood 106, 3888-3894 (2005).

37. Jouault, T. et al. Candida albicans phospholipomannan is sensed through toll-like receptors. J. Infect. Dis. 188, 165-172 (2003).

38. Jouault, T. et al. Specific recognition of Candida albicans by macrophages requires galectin-3 to discriminate Saccharomyces cerevisiae and needs association with TLR2 for signaling. J. Immunol. 177, 4679-4687 (2006).

39. Dillon, S. et al. Yeast zymosan, a stimulus for TLR2 and dectin-1, induces regulatory antigen-presenting cells and immunological tolerance. J. Clin. Invest. 116, 916-928 (2006).

40. Gantner, B.N., Simmons, R.M., Canavera, S.J., Akira, S. \& Underhill, D.M. Collaborative induction of inflammatory responses by dectin-1 and Toll-like receptor 2. J. Exp. Med. 197, 1107-1117 (2003).

41. Coste, A. et al. IL-13 attenuates gastrointestinal candidiasis in normal and immunodeficient RAG-2(-/-) mice via peroxisome proliferator-activated receptor-gamma activation. J. Immunol. 180, 4939-4947 (2008).

42. Coste, A. et al. PPARgamma promotes mannose receptor gene expression in murine macrophages and contributes to the induction of this receptor by IL-13. Immunity 19, 329-339 (2003).

43. Gillum, A.M., Tsay, E.Y. \& Kirsch, D.R. Isolation of the Candida albicans gene for orotidine-5'-phosphate decarboxylase by complementation of S. cerevisiae ura3 and E. coli pyrF mutations. Mol. Gen. Genet. 198, 179182 (1984).

44. Siegmund, B. et al. Adenosine kinase inhibitor GP515 improves experimental colitis in mice. J. Pharmacol. Exp. Ther. 296, 99-105 (2001)

45. Jawhara, S. et al. Murine model of dextran sulfate sodium-induced colitis reveals Candida glabrata virulence and contribution of beta-mannosyltransferases. J. Biol. Chem. 287, 11313-11324 (2012).

46. Saviozzi, S., Cordero, F., Lo lacono, M., Novello, S., Scagliotti, G.V. \& Calogero, R.A. Selection of suitable reference genes for accurate normalization of gene expression profile studies in non-small cell lung cancer. BMC Cancer 6, 200 (2006). 